\title{
Relevant responses in grape cells exposed to endophytic fungi or to fungal extracts at transcriptome and metabolome scopes
}

Jin-Zhuo Qu

Yunnan University

Fang Liu

Yunnan University

Xiao-Xia Pan

Yunnan University

Yin-Min Ma

Yunnan University

Ming Zhou

Yunnan University

Si-Yu Xiang

Yunnan University

Chang-Mei Liao

Yunnan University

Tong Li

Yunnan University

Han-Bo Zhang

Yunnan University

Ming-Zhi Yang ( $\nabla$ yangmzh@ynu.edu.cn )

Yunnan University https://orcid.org/0000-0002-3134-7643

Research article

Keywords: grape cells, relevant response, fungal extracts, living endophytes, omics scope, crop management, eco-safety promise

Posted Date: May 10th, 2020

DOI: https://doi.org/10.21203/rs.3.rs-24847/v1

License: (c) (1) This work is licensed under a Creative Commons Attribution 4.0 International License. Read Full License 


\section{Abstract}

Background: Endophytes with beneficial effects on hosts are potentially utilized in crop managements. However, the use of living endophytes will risk the possible pathogenicity or other ecological problems. Whereby, it will be great value if functions of certain endophytes on crops could be replaced by elicitors extracted from living endophytes. For addressing this issue, the present work therefore compared the impacts of three endophytic fungal strains and their soluble extracts on grape cells at both the transcriptomic and metabolome scopes.

Results: Our results revealed that the exposure to living fungi or fungal extracts triggered responses in terms of both the differentially expressed genes (DEGs) and the differentially regulated metabolites (DRMs) in grape cells, with great fungal strain-specificities. Compared to the living fungi exposed grape cells, less DEGs and DRMs responses were initiated in most of the corresponding fungal extracts treated grape cells. Nevertheless, major proportions of specific DEGs, DRMs, and the subsequently enriched GO function terms and KEGG pathways in living fungi treated grape cells were also detected in those fungal extracts exposed grape cells.

Conclusions: The work demonstrated that the specific effects of certain living endophytic fungus on grape cells could be replaced or partially replaced by their fungal extracts, and confirmed an alternative approach to utilize endophytes in agriculture with more eco-safety promises.

\section{Background}

Endophyte was defined as all microorganisms colonize internal plant tissues for all or part of their lifetime [1]. The groups of endophytes within host plants represents a hot topic of great relevance due to the multiple standpoints from which it can be approached and considered [2]. Functions of endophytes to host plants ranged from mutualistic to pathogenic, with most of them in plant may appear neutral effects or no function at all to their hosts, namely "commensal" [1]. Even though, the beneficial interactions of endophytes with their hosts still attracted great attentions for their possible utilization in crop quality managements, especially to those perennial woody crops such as grapevines, which the hosts could receive long term effects [2]. Several endophytes with positive functions such as adapt to abiotic stresses, defense against pathogens, growth promotion and plant quality improvements on grapevines have been revealed and developed [2]. These endophytes as bacterial endophyte Paraburkholderia phytofirmans strain PSJN has been observed to be conducive as a chilling stress-preventive trait as plants bearing this endophyte $[3,4]$. The antifungal activity against downy mildew coursing pathogen Plasmopara viticola of diketopiperazines (DKPs) produced by an endophytic strain of Alternaria. alternata isolated from grapevine leaves showing anomalous downy mildew symptoms [5]. And inoculation of endophytic fungal strains such as CXB-11 (Nigrospora sp.) and CXC-13 (Fusarium sp.)," exerting significant effects on grapevine metabolites [6]. Additionally, fungal strains could specifically introduce novel metabolites into grape cells during a dual culture experiment, and these results implicated the possible of applying endophytes to improve physiologically and biochemically the qualities of 
grapevines [7]. Obviously, the application of endophytes in crop managements could directly as living endophytes or elicitors extracted from them. However, the effects associations and differences between living and the corresponding extracts of endophytes on host plant cells are fundamentally before endophytic elicitors could be successfully applied.

Generally, the use of living endophytes and inactive endophytic extracts both have its own advantages and disadvantages. The inoculation of living endophytes always expect to receive long term and stable effects to their hosts. However, this will risk the used endophytes may sometimes be turned into pathogens in certain circumstances or even cause further unexpected ecological problems. A great deal of endophytes which isolated from healthy plants have proved the pathogens to their host or other plants [1]. In addition, researches have revealed that most of the purposely introduced endophytes into certain

plant or plant rhizosphere always disappeared within times [8]. On the other side, the advantages of using endophytic extracts or elicitors could be purposely designed in proper times and dosages, without concerning the risk of turning into pathogens. However, the premise is that the inactive agents from certain endophytes should still produce similar effects to the host plants as the living endophytes did. The present research was concerned this issue by comparing the responses in in vitro grape cells to living endophytic fungi and their extracts at transcriptome and metabolome scopes.

\section{Results}

The transcriptome and metabolome analyses to all samples were reached the basic standards of quality control. An average 50 million clean reads from each sample were acquired with Q30 >93\%. And more than $93 \%$ acquired clean reads of each sample library were successfully mapped in the reference genomes (Additional file 1).

Principal components analyses (PCA) weakly resolved the transcriptome and the metabolome profiles in grape cell samples which treated with different endophytic fungal strains or their corresponding extracts (Fig. 1). Replicates of one treatment could almost grouped together in PCA plots based on either transcripts or metabolites profiles in grape cells (Fig. 1). For PCA of transcriptome, PC1 could well resolve sample groups $\mathrm{C} 11$ (grape cells treated with endophytic fungal strain C11, and the same of R12 and R32) and R32 with other sample groups except group R12E (grape cells treated with extract of endophytic fungal strain R12, and the same as C11E and R32E). Whereas, PC2 obviously resolved group R32 from other groups except group R32E. Group R12 was closely joined to the group C11E and these groups together could be divided from the groups R32E and R12, in this PCA analysis (Fig. 1 left). Groups R32E and C11E closely joined to the control group in transcriptome PCA plot, due to their similar transcripts profiles (Fig. 1 left). For metabolome assay, a group of mixed samples was randomly added as quality control (QC) during the process of analysis, and all replicates of these QC samples were well clustered together, and located almost within the middle of other sample groups, implicated the validation of the metabolome analysis (Fig. 1 right). In the metabolome PCA, PC1 could resolve group $\mathrm{C} 11$ with other groups, while PC2 resolved groups R12, R12E from groups C11, R32 and other groups (Fig. 1 right). Besides, sample groups R12 and R12E were observed close together in PCA plots of both transcriptome 
and metabolome analysis (Fig. 1). Similar to the transcriptomic assay, groups R32E and C11E were also closely joined to the control group in the PCA of metabolome (Fig. 1 right).

As have compared to the corresponding controls, the counts of differentially expressed genes (DEGs,) and differentially regulated metabolites (DRMs) were summarized in Figure 2. For most of the treatments, more counts of DEGs and DRMs were triggered in living fungi exposed grape cells than that of their corresponding fungal extracts treated grape cells (Fig. 2). For living fungi treatments, fungal strains initiated counts of DEGs in grape cells from the most to the least were C11 > R32 > R12 in transcriptomic analysis (Fig. 2 A-C). Whereas in metabolome assay, the used living fungal strains triggered DRMs counts in grape cells from the most to the least were R12>C11>R32 (Fig. $2 \mathrm{E}-\mathrm{F}$ ). Regardless the treatments with living fungi or fungal extracts in transcriptome analysis, more proportions of DEGs in grape cells were down regulated (Fig. 2 A-C). For metabolome assay, all living fungi treatments exclusively initiated greater counts of DRMs in grape cells than those triggered by fungal extracts (Fig. 2 D-F). And those living fungi treatments also tend to cause more proportions of down regulated DRMs in grape cells (Fig. 2 D-F).

The exposure to different living fungi and soluble extracts from different fungal strains resulted in different proportions of specific responses in DEGs and DRMs counts (Fig. 3). In transcriptome assay, dual culture with fungal strains $\mathrm{C} 11, \mathrm{R} 12$ and $\mathrm{R} 32$ initiated $49.5 \%, 28.1 \%$ and $44.7 \%$, respectively the specific DEGs counts in grape cells. And the proportions of co-detected DEG counts in these fungal strains treated grape cells were $8.7 \%, 29.7 \%$ and $10.9 \%$, respectively. Beside the treatment of R12, treatments with other two fungal strains $\mathrm{C} 11$ and R32 initiated smaller proportions of co-detected DEGs than that of the specific ones(Fig. 3 up-left). As for fungal extract treatments, the proportions of specific DEGs in C11E, R12E and R32E treated grape cells were $57.4 \%, 75.6 \%$ and $27.0 \%$, respectively, while the proportions of the co-detected DEGs in these fungal extracts exposed grape cells were only $12.8 \%, 1.2 \%$ and $3.9 \%$, respectively (Fig. 3 up-right). And in metabolome analysis, exposure to fungal strains C11, R12 and R32 caused $44.4 \%, 48.8 \%$ and $30.8 \%$, respectively the specific DRMs in grape cells, and the proportions of specific DRMs in C11E, R12E and R32E treated grape cells were $11.1 \%, 60.7 \%$ and $28.6 \%$, respectively (Fig. 3 below). The exposure to fungal extracts C11E and R32E initiated greater proportions ( $77.8 \%$ and $50 \%$ respectively) of co-detected DRMs in grape cells (Fig. 3 ). Whenever in transcriptome or in metabolome assays, R12E were all triggered greatest proportions of specific responses of DEGs and DRMs counts in grape cells (Fig. 3).

However, when concerning the treatment pairs (one treatment pair was defined as the treatments of living fungus and the corresponding fungal extract), major proportions of responses of DEGs and DRMs in fungal extracts exposed grape cells were encompassed within the corresponding living fungi triggered DEGs and DRMs in grape cells, with the only exception of treatment pair R12 and R12E in transcriptomic assay (Fig. 4). In transcriptome analysis, in total 70.2 \% and 73.7\% DEGs in C11E and R32E treated grape cells were detected respectively, within the C11 and R32 treated grape cells (Fig. 4). One exception was that only $22.2 \%$ DEGs in R12E treated grape cells were co-detected in the corresponding living fungus (R12) treated grape cells (Fig. 4). Meanwhile, 88.9\%, 75\% and 71.4\% DRMs in C11E, R12E and R32E 
treated grape cells, were detected simultaneously in C11, R12 and R32 dual cultured grape cells respectively, in the metabolome analysis (Fig. 4).

All those significant DEGs were subjected to Gene ontology (GO) and Kyoto Encyclopedia of Genes and Genomes (KEGG) enrichment analysis. The significantly enriched GO function terms and KEGG pathways in grape cells which initiated by fungi and fungal extracts were greatly dependent upon the used endophytic fungal strains (Fig. 5). For treatment pair C11 and C11E, the exposure to living fungal strain C11 significantly enriched more GO function terms in the biological processes (BP), cellular components (CC) and molecular functions (MF) in grape cells, while in C11E treated grape cells enriched almost no significant GO terms in BP and CC functions (Fig. 5). However, GO terms in MF which associate the functions of DNA binding transcription factor activity (DBTFA), transcription regulator activity (TRA) and oxidoreductase activity (ORA) in C11E treated grape cells were significantly enriched, and these GO terms were also significantly enriched in $\mathrm{C} 11$ exposed grape cells (Fig. 5). Besides, GO terms in MF category of DBTFA and TRA were also detected the significant enrichments in R12E and R32 treated grape cells (Fig. 5). Different to the treatment pair $\mathrm{C} 11$ and $\mathrm{C} 11 \mathrm{E}$, the R12 exposure almost caused no significant $\mathrm{GO}$ term enrichment in grape cells, while several GO terms in categories BP, CC and MF were significantly enriched in R12E exposed grape cells (Fig. 5). For treatment pair R32 and R32E became another story. Living fungal strain R32 dual cultured grape cells enriched great amounts of significant GO terms in categories of BP, CC and MF, and some of the significantly enriched GO terms in BP and MF were also detected the enrichment in R32E treated grape cells (Fig. 5).

In KEGG enrichment analysis, the significant enriched KEGG signal pathways in this experiment were fell into plant hormone signal transduction, photosynthesis, cyanoamino acid metabolism, flavonoid biosynthesis, plant Circadian rhythm, pentose and glucoronate interconversions, phenylpropanoid biosynthesis and plant-pathogen interaction(Fig. 6). And KEGG pathways of plant hormone signal transduction and photosynthesis were significant enriched in grape cells of most the treatments in this experiment (Fig. 6). Nevertheless, most of the enriched KEGG pathways in grape cells which enriched in fungal extracts treated grape cells were also comprised within their corresponding living fungi exposed grape cells (Fig. 6). However, the significant enriched KEGG pathways in fungi and fungal extracts exposed grape cells were greatly varied from one fungal strain to another (Fig. 6).

As comparing the enriched GO terms and KEGG pathways in those treatment pairs, almost all those enriched GO terms and KEGG pathways in C11E and R32E exposed grape cells were included within those of the corresponding fungal strains $\mathrm{C} 11$ and R32 treated grape cells (Fig. 7). Even the treatment pair R12E and R12, major proportions of enriched GO terms and KEGG pathways $(47.4 \%$ and $76.1 \%$, respectively) in R12E treated grape cells were comprised in that of the R12 exposed grape cells (Fig.7), regardless the lowest (22.2\%) co-detected DEGs in R12E and R12 treated grape cells (Fig. 3).

DEGs which involved in significant co-enriched GO terms and KEGG pathways were summarized in Table 1 and Table 2, respectively. The more or less significantly enriched specific GO terms or KEGG pathways in living fungal treated grape cells could also be significantly enriched in the corresponding fungal 
extracts treated grape cells (Table 1 and Table 2). Such enriched specific GO terms as G0:0008017 (microtubule binding), G0:0015631(tubulin binding) and G0:0046527 (glucosyltransferase activity) in R12 and R12E treated grape cells, and G0:0005618 (cell wall), G0:0005576 (extracellular region), G0:0030312 (external encapsulating structure) and G0:0071944 (cell periphery) in R32 and R32E exposed grape cells (Table 1). As well as the significant enriched fungal strain-specific KEGG pathways as vvi04016 (MAPK signaling pathway-plant) in C11/C11E, vi03030 (DNA replication) in R12/R12E and vvi04626 (Plant-pathogen interaction) in R32/R32E treated grape cells, respectively (Table 2). Beside the treatment pair R12 and R12E, more counts of DEGs which involved in certain GO function terms or KEGG pathways were enriched in living fungi treated grape cells than that of the fungal extracts exposed grape cells (Table 1 and Table 2). However, despite the differences of total counts of DEGs enriched in certain GO terms or KEGG pathways, the ratio of up/down regulated DEGs in certain GO terms or KEGG pathways maintained a similar trends between living fungi and the corresponding fungal extracts treated grape cells (Table 1 and Table 2).

Table 1. Comparison of the differentially expressed genes (DEGs) involved in the significant co-enriched gene ontology (GO) terms in living fungi and fungal extracts treated grape cells

\begin{tabular}{lllllll}
\hline GO ID & $\begin{array}{l}\text { All } \\
\text { counts }\end{array}$ & $\begin{array}{l}\text { Up } \\
\text { regulated }\end{array}$ & $\begin{array}{l}\text { Down } \\
\text { regulated }\end{array}$ & $\begin{array}{l}\text { All } \\
\text { counts }\end{array}$ & $\begin{array}{l}\text { Up } \\
\text { regulated }\end{array}$ & $\begin{array}{l}\text { Down } \\
\text { regulated }\end{array}$ \\
\hline GO:0003700 & 102 & 19 & 83 & 35 & 1 & 34 \\
\hline GO:0140110 & 104 & 19 & 85 & 35 & 1 & 34 \\
\hline GO:0048046 & 10 & 1 & 9 & $\begin{array}{l}\text { R12E } \\
13\end{array}$ & 0 & 13 \\
\hline GO:0003700 & 43 & 11 & 32 & 80 & 9 & 71 \\
\hline GO:0008017 & 12 & 12 & 0 & 20 & 20 & 0 \\
\hline GO:0140110 & 44 & 11 & 33 & 82 & 9 & 73 \\
\hline GO:0015631 & 12 & 12 & 0 & 20 & 20 & 0 \\
\hline GO:0016762 & 10 & 1 & 9 & 13 & 0 & 13 \\
\hline GO:0046527 & 17 & 3 & 14 & 24 & 4 & 20 \\
\hline GO:0048046 & 17 & 1 & 16 & 6 & 0 & 6 \\
\hline GO:0005618 & 24 & 1 & 23 & 7 & 0 & 7 \\
\hline GO:0005576 & 17 & 1 & 16 & 6 & 0 & 6 \\
\hline GO:0030312 & 24 & 1 & 23 & 7 & 0 & 7 \\
\hline GO:0071944 & 30 & 3 & 27 & 10 & 0 & 6 \\
\hline GO:0003700 & 74 & 16 & 58 & 33 & 2 & 31 \\
\hline GO:0140110 & 75 & 16 & 59 & 33 & 2 & 31 \\
\hline GO:0016762 & 17 & 1 & 16 & 6 & 0 & 6 \\
\hline
\end{tabular}

Table 2. Comparison of the differentially expressed genes (DEGs) involved in the significant coenriched Kyoto Encyclopedia of Genes and Genomes (KEGG) pathways in living fungi and fungal extracts treated grape cells 


\begin{tabular}{|c|c|c|c|c|c|c|}
\hline KEGG ID & $\begin{array}{l}\text { All } \\
\text { counts }\end{array}$ & $\begin{array}{l}\text { Up } \\
\text { regulated }\end{array}$ & $\begin{array}{l}\text { Down } \\
\text { regulated }\end{array}$ & $\begin{array}{l}\text { All } \\
\text { counts }\end{array}$ & $\begin{array}{l}\text { Up } \\
\text { regulated }\end{array}$ & $\begin{array}{l}\text { Down } \\
\text { regulated }\end{array}$ \\
\hline & C11 & & & C11E & & \\
\hline vvi04075 & 72 & 23 & 49 & 24 & 4 & 20 \\
\hline \multirow[t]{2}{*}{ vvi04016 } & 37 & 15 & 22 & 14 & 4 & 10 \\
\hline & R12 & & & R12E & & \\
\hline vvi04075 & 31 & 6 & 25 & 50 & 13 & 37 \\
\hline \multirow[t]{2}{*}{ vvi03030 } & 11 & 11 & 0 & 17 & 17 & 0 \\
\hline & R32 & & & R32E & & \\
\hline vvi04075 & 45 & 19 & 26 & 10 & 5 & 5 \\
\hline vvi04626 & 33 & 11 & 22 & 11 & 2 & 9 \\
\hline
\end{tabular}

In metabolome analysis, DRMs in different fungi and fungal extracts treated grape cells were also performed the KEGG analyses. Dual cultivation with living fungi C11, R12 and R32 enriched 18, 2 and 3 specific KEGG pathways, respectively in grape cells, with $12 \mathrm{KEGG}$ pathways which have co-enriched in all these fungi treated grape cells (Fig. 8). For the treatments of fungal extracts from different fungal strains, 5 and 3 specific KEGG pathways were enriched in R12E and R32E exposed grape cells. No significant specific KEGG pathways were enriched in C11E treated grape cells. And as concerning the treatment pairs however, 100\%, 73.3\% and 92.3\% KEGG pathways enriched in C11E, R12E and R32E treated grape cells were enriched simultaneously in C11, R12 and R32 treated grape cells (Fig. 8).

Table 3 listed the differential regulated metabolites (DRMs) in the metabolome analysis with the threshold as Log2 (Fold. Change) $\geq 1.0$, and VIP $\geq 1.0$ (Table 3). The exposure to fungus $\mathrm{C} 11$ and its extracts more possibly influenced metabolites in compound class of amino acids and derivatives, while exposed to fungus R12 and its extract (R12E) initiated more metabolites changes in classes of glycerol ester, lysophosphatidylcholine (LPC) and lysophosphatidyl ethanolamine (LPE) (Table 3). And other classes of metabolites could be influenced more or less by most of the treatments in this metabolome assay (Table 3). In most cases, co-regulated metabolites in living fungi or the corresponding fungal extracts treated grape cells maintained the same trends of up or down regulations. It deserve to note that the metabolite $\varepsilon$-Viniferin was up regulated in living fungi treated grape cells, while this compound was greatly down regulated in fungal extracts treated grape cells (Table 3 ).

Table 3. Significant regulated metabolites in grape cells after the treatment with different endophytic fungi and fungal extracts. 


\begin{tabular}{|c|c|c|c|c|c|c|c|}
\hline \multirow[b]{2}{*}{ Class $^{a}$} & \multirow[b]{2}{*}{ Significant changed compounds } & \multicolumn{6}{|c|}{ Log (Fold change) } \\
\hline & & C11 & $\mathrm{C} 11 \mathrm{E}$ & R12 & R12E & R32 & R32E \\
\hline Alkaloids & Caffeine & & & -1.12 & & -10.9 & -1.09 \\
\hline \multirow[t]{18}{*}{ AA } & 5-Aminovaleric acid & -1.30 & & & & & \\
\hline & Trans-4-Hydroxy-L-proline & -1.15 & & & & & \\
\hline & L-Tyramine & -3.16 & -1.65 & -2.17 & -4.06 & -4.07 & -14.68 \\
\hline & 1,2-N-Methylpipecolic acid & -1.18 & & & & & -1.01 \\
\hline & N-Acetyl-L-leucine & 2.43 & & & & & \\
\hline & N-Acetylaspartate & 1.40 & & & & & \\
\hline & N-Acetyl-L-glutamic acid & 1.23 & & & & & \\
\hline & N-Acetylmethionine & -1.79 & -1.28 & -1.30 & -1.87 & -2.84 & -4.95 \\
\hline & N-(3-Indolylacetyl)-L-alanine & & & 1.40 & & & \\
\hline & L-Homocystine & & & -1.04 & & & \\
\hline & Leucylphenylalanine & & & -1.24 & & & \\
\hline & L-Glutamic acid O-glycoside & & & & -2.98 & -4.31 & \\
\hline & N- $\alpha$-Acetyl-L-arginine & 2.02 & & & & & \\
\hline & N-Acetyl-L-tyrosine & 4.55 & & & & & \\
\hline & Lysine butyrate & -1.60 & -1.18 & & & & \\
\hline & N-(3-Indolylacetyl)-L-alanine & 3.40 & & & & & \\
\hline & Leucylphenylalanine & -1.30 & & & & & \\
\hline & L-Glutaminyl-L-valyl-L-valyl-L-cysteine & 8.90 & & 8.34 & 8.82 & 1.01 & \\
\hline Anthraquinone & 6-Hydroxyrumicin-8-O-D-glucopyranoside & 1.85 & & & & 1.01 & \\
\hline Coumarins & Skimmin & 1.82 & & & & 1.23 & \\
\hline \multirow[t]{2}{*}{ Dihydroflavone } & Eriodictyol 7-O-glucoside & & & 1.60 & 1.12 & & \\
\hline & Hesperetin 7-O-neohesperidoside(Neohesperidin) & -15.6 & -2.09 & -6.64 & -15.6 & & -15.6 \\
\hline Flavonols & Isorhamnetin-3-O- $\beta$-D-glucoside & & & 1.24 & & & \\
\hline \multirow[t]{3}{*}{ FFA } & 13-HOTrE(r) & & & 1.06 & & & \\
\hline & $\gamma$-Linolenic Acid & & & & -1.02 & & \\
\hline & 11-Octadecanoic acid(Vaccenic acid) & 1.40 & & & & & \\
\hline Glycerol ester & 1- $\alpha$-Linolenoyl-glycerol* & & & -1.00 & & & \\
\hline \multirow[t]{4}{*}{ LPC } & LysoPC 18:1 & & & 1.13 & & & \\
\hline & LysoPC 18:1(2n isomer) & & & 1.23 & & & \\
\hline & LysoPC 18:0 & & & 1.01 & 1.11 & & \\
\hline & LysoPC 18:0(2n isomer) & & & & 1.03 & & \\
\hline \multirow[t]{7}{*}{ LPE } & LysoPE 16:0 & & & 1.89 & 1.74 & & \\
\hline & LysoPE 16:0(2n isomer) & & & 1.63 & 1.73 & & \\
\hline & LysoPE 18:3 & & & & & -9.51 & \\
\hline & LysoPE 18:2 & & & 1.56 & 1.46 & & \\
\hline & LysoPE 18:2(2n isomer) & & & 1.61 & 1.29 & & \\
\hline & LysoPE 18:1 & & & 1.90 & 1.54 & -8.57 & \\
\hline & LysoPE 18:1(2n isomer) & & & 1.48 & 1.10 & & \\
\hline
\end{tabular}




\begin{tabular}{|c|c|c|c|c|c|c|c|}
\hline \multirow[b]{2}{*}{ Class } & \multirow[b]{2}{*}{ Significant changed Compounds } & \multicolumn{6}{|c|}{ Log (Fold Change) } \\
\hline & & C11 & $\mathrm{C} 11 \mathrm{E}$ & R12 & R12E & R32 & R32E \\
\hline \multirow[t]{3}{*}{ ND } & 5-Methylcytosine & -1.40 & & & & & \\
\hline & Xanthine & -1.22 & & & & & \\
\hline & 9-( $\beta$-D-Arabinofuranosyl)hypoxanthine & & & 1.01 & & & \\
\hline \multirow[t]{5}{*}{ Organic acids } & 6-Aminocaproic acid & -1.21 & & & & & \\
\hline & 2-Furanoic acid & & & & & -1.49 & \\
\hline & 3-Hydroxy-3-methyl butyric acid & & & & & 1.06 & \\
\hline & Diethyl phosphate & & & -1.51 & & & \\
\hline & 3-Hydroxyanthranilic acid & -9.61 & & & & & \\
\hline \multirow[t]{3}{*}{ Others } & Octadecenoic amide & & & 2.05 & & & \\
\hline & Propyl2-(trimethylammonio)ethyl phosphate & 1.52 & & 1.68 & & & \\
\hline & $\alpha$-Viniferin & -11.2 & -1.67 & 2.03 & -11.2 & -1.52 & -2.47 \\
\hline $\mathrm{PC}$ & Choline alfoscerate & & & & & & -2.25 \\
\hline \multirow[t]{13}{*}{ PA } & 3,4-Dihydroxybenzeneacetic acid & & & 1.15 & & & \\
\hline & Trans-4-Hydroxycinnamic Acid Methyl Ester & & & -1.24 & & & \\
\hline & 3,4-Dihydroxybenzeneacetic acid & 1.21 & & & & & \\
\hline & Methyl ferulate & 1.53 & & & 1.18 & 1.44 & 1.78 \\
\hline & Isosalicylic acid O-glycoside & & & & & 1.32 & \\
\hline & 3-Hydroxy-5-Methylphenol-1-Oxy- $\beta$-D-Glucose & & & & 1.04 & & \\
\hline & Feruloylmalic acid & 1.87 & & 1.56 & 1.16 & 2.78 & 1.81 \\
\hline & 3-Hydroxy-4-isopropylbenzylalcohol 3-glucoside & & 1.07 & 1.04 & 1.15 & 1.61 & \\
\hline & 1'-O-Vanilloyl- $\beta$-D-glucoside & & & & 1.14 & & \\
\hline & Syringic acid O-glucoside & & & -1.06 & & 1.08 & \\
\hline & Trihydroxycinnamoylquinic acid & 1.08 & & -9.22 & & 1.61 & -9.22 \\
\hline & Syringin & & & 1.18 & 1.14 & 1.95 & \\
\hline & p-Coumaroylcaffeoyltartaric acid & 3.32 & & & & 1.57 & \\
\hline Plumerane & Indole & -1.11 & & & & & \\
\hline \multirow[t]{3}{*}{ SA } & D-Glucoronic acid & -1.73 & & & -1.18 & & \\
\hline & D-(+)-Melezitose* & & & 1.28 & & & \\
\hline & $\mathrm{D}(+)$-Melezitose O-rhamnoside & 1.38 & & 1.38 & & & \\
\hline \multirow[t]{3}{*}{ Stilbene } & Piceid & 1.31 & & 1.70 & 1.63 & 2.03 & \\
\hline & $\varepsilon$-Viniferin & 1.25 & -9.22 & 2.61 & -9.22 & & -9.22 \\
\hline & Resveratrol-O-diglucoside & & & & & -1.25 & \\
\hline Tannin & 2-O-Galloyl- $\beta$-D-glucose & & & -1.80 & 1.93 & 1.23 & -1.03 \\
\hline \multirow[t]{2}{*}{ Triterpene } & Camaldulenic acid & -10.9 & 1.28 & -10.9 & -10.9 & -10.9 & -10.9 \\
\hline & 2-Hydroxyoleanolic acid & -2.78 & 1.34 & -3.05 & -3.06 & -1.82 & -3.59 \\
\hline \multirow[t]{2}{*}{ Vitamin } & Nicotinamide & & & & & -1.01 & \\
\hline & Pyridoxine & & & 1.01 & & & \\
\hline
\end{tabular}

AA: Amino acids and derivatives; FFA: Free fatty acid; LPC: Lysophosphatidylcholine; LPE: Lysophosphatidyl ethanolamine; ND: Nucleotides and derivatives; PC: Choline alfoscerate; PA: Phenolic acids; SA: Saccharides and Alcohols.

\section{Discussion}

Endophytes have been greatly concerned due to their beneficial interactions with host plants, such as growth promoting, stress adaptability, biochemistry shaping and others [1, 2]. And these hyperdiverse communities of endophytes provided tremendous resources for crop managements as multiple 
standpoint. This will great interest to those crops given organoleptic sensitive products, such as grapevines, in which a fine-turned metabolic changes might trigger obvious quality and characteristic effects on the resultant wine $[2,6]$. Researches have covered the metabolic impacts of pure cultured fungal endophytes on grape cells under well controlled conditions in dual cultures, which appeared fungal strain, species and other taxonomical specificities in shaping the metabolites of grape cells $[7,9$, 10]. And the specific shaping of fungal strains on grape cells metabolism were furtherly confirmed in this research at both the transcriptomic and metabolomic scopes (Fig.1, Fig. 3). On the other side, elicitors extracted from fungal or bacterial endophytes were continuously revealed the functions in initiating varies physiological and biochemical responses in host plants or cells [11-13]. However, these experiments concerned the impacts of endophytes and elicitors from them on host plants were all separately carried. One study reported recently that dual culture with fungal endophytes could quantitatively and compositionally modify the anthocyanins in grape cells, and their fungal extracts conferred the similar effects as well [14]. Could this be hypothesized that endophytic extracts maintained some specific effects or functions on host plant as the living endophyte had? The present work therefore proposed methods covered this question by comparing the underlying associations and differences of responses between plant cells which have exposed separately with living endophytes or their soluble extracts, at the transcriptome and metabolome scopes.

Technologies of RNA-Sequencing and metabolites profiling provide sufficient information to monitor the responses in grape cells under treatments. The exposure to Living fungi and the fungal extracts all resulted to the creation of differential gene transcripts and metabolites profiles in grape cells (Fig. 1). Treatment groups could be better resolved the transcriptomic results than that of the metabolome results in the PCA, whereas the relative layouts and distances amongst groups were quit similar between PCA plots based on the data of transcriptome and metabolome analysis (Fig. 1). These results implicated a better approach with more sensitivity of using transcriptome in detecting the differential responses in grape cells under treatments. The relative isolated layout of treatment group pairs of C11 /C11E and R32/R32E in both PCA plots (Fig. 1), indicated the differences of transcriptional and metabolic impacts on grape cells between treatments of living fungi and fungal extracts. While treatment group R12/R12E treated grape cells conferred a relatively higher degrees of identities of metabolites profiles, due to their closely gather in the PCA plots. Treatment groups which closer to the control groups in PCA plots such as C11E and R32E (Fig. 1), represent their less impacts on both the transcripts and metabolites profiles in grape cells (Fig. 1). And this conclusion was repeatedly proved in the following results, due to the less detected DEGs and DRMs in C11E and R32E treated grape cells (Fig. 2 and 3; Fig. 5 and 6; Table 1 and 2). In fact, greater responses of grape cells to living fungi than to fungal extracts were observed as evaluated in DEGs and DRMs counts, as wells as the significant enriched GO terms and KEGG pathways, with few exceptions (Fig. 2, Fig. 4-6). Another characteristic of differences in grape cells between responses against fungi and fungal extracts was that the fungi triggered DEGs in grape cells could always cover great ranges of GO terms or KEGG pathways, whereas fungal extracts tend to the enrichment of some specific GO terms or KEGG pathways (Fig. 5 and Fig. 6). Fungal elicitors were always reported applied to initiate certain specific metabolism pathways or certain useful metabolites in plant cells [11, 15-17]. 
Results of this research fully demonstrated the differences of grape cells in responding to the living fungi and their fungal extracts, as well as the treatments with different fungal strains and different fungal extracts. And the more or less specific DEGs and DRMs in grape cells were detected in different living endophytic fungal strains or different fungal extracts exposed grape cells (Fig. 4), further implicated the fungal strain dependent responses of grape cells against both the living fungi and fungal extracts.

However, despite the different responses of grape cells against the fungi and their extracts, obvious relevance were detected underlying the interactions between grape cells exposed to living fungus and to the corresponding fungal. Firstly, major proportions of fungal extracts triggered DEGs and DRMs in grape cells were comprised inside the corresponding living fungi caused DEGs and DRMs in grape cells (Fig. 3). Secondly, major proportions of the enriched GO terms and KEGG pathways in certain fungal extract treated grape cells were also encompassed within those of the corresponding living fungus exposed grape cells, including some significantly enriched fungal strain-specific GO terms or KEGG pathways (Fig. 5, Fig. 6, Table 1 and Table2). And the ratios of up/down regulated DEGs involved in each co-enriched GO term or KEGG pathway were maintained similar trends between living fungi and their fungal extracts treated grape cells (Table 1 and Table 2). Finally, most of those co-detected DRMs in fungi and fungal extracts treated grape cells also maintained the same trends of up or down regulations, with few exceptions (Table 2). Therefore, at the scopes of transcriptome and metabolome, obvious relevance were observed between responses in grape cells exposed to fungi and to their extracts. In another word, fungal elicitors or extracts could maintain partially the specific effects as their living fungi on the host plants. And this implicated that instead of living fungal agents, applying elicitors or extracts from certain fungus could also receive the similar effects as their living fungi in plant managements, with the advantages of the dosage and timing of application could be well under controlled. And most important, to apply inactive elicitors could avoid the risks of other eco-problems such as the used fungi conditionally turned into pathogens.

In present research, we used water soluble extracts, which contained not only the specific elicitors of the used fungus, but also a great deal of other general soluble components which are widely consisted in most of the fungi. Whereby, with purposely prepared specific extracts or elicitors, one could filter those unexpected metabolism impacts with more emphasizing the effects we most concerned. However, for reaching a satisfied purpose of using certain fungal elicitors, methods of fungal mycelium extraction, dosage and time of application should be all investigated ahead.

\section{Conclusions}

Endophytic fungi and their soluble extracts could all cause fungus-specific metabolic responses in grape cells at transcriptomic and metabolomic scopes. The living endophytic fungi initiated greater responses in terms of differentially expressed genes (DEGs) and differentially regulated metabolites (DRM) in grape cells than those of their corresponding fungal extracts. Fungal extracts could maintain proportions of specific effects as their corresponding living fungi did on grape cells. And the work confirmed that the 
effects of living fungi on grape cells could be replaced or partially replaced by their extracts with great eco-safety promises.

\section{Methods}

Preparation of grape cells: A cell line (CBL, kindly provided by Professor Serge Delrot, Lab. of Grapevine Physio-Ecological and Functional Genomics, France) which induced from the flesh of grape berries (Vitis vinifera, cultivar: Cabernet sauvignon) was used in this study. B5 solution with $3 \%$ sucrose, $0.2 \mathrm{mg} / \mathrm{L}$ cytokinin, $0.1 \mathrm{mg} / \mathrm{L}$ naphthylacetic acid (NAA) and $0.8 \%$ agar (B5 agar medium) was prepared as the medium for callus sub-culture as well as the subsequent treatments with fungi or fungal extracts. The grape calli prepared for the experiment were in the logarithmic growth phase.

Preparation of endophytic fungal strains and fungal soluble extracts: Three endophytic fungal strains C11 (Fusarium sp.), R12 (Niqrospora sphaerica) and R32 (Alternaria alternaria),, were used in this experiment, which isolated previously from grapevine leaves from local vineyards in Yunnan province, China. Living Fungi used to establish dual culture with grape cells were firstly cultured in PDA plates for one week, and fungal mycelia discs generated with a $0.5 \mathrm{~cm}$ punch were used in the dual cultivation. For preparing fungal extracts, fresh fungal mycelia were collected via filtration from suspension cultures. After several washes with distilled water, fungal mycelia were first weighed and then ground with a certain volume of distilled water, followed by extraction in an ultrasonic cleaner for 30 minutes. The mixtures were subsequently centrifuged at $8000 \mathrm{rpm}$ for 15 minutes, and the supernatant was preserved at $4{ }^{\circ} \mathrm{C}$ as the fungal extract for future use.

Treatments of grape cells with living fungi or fungal extracts: The solid dual culture systems were established according to Huang et al. (2017), with some modifications. Instead of inoculating grape calli into the middle of petri dishes, grape calli were inoculated into one side of B5 plates, and fungal mycelium discs were then inoculated on opposite side of plates 5 days after the inoculation of grape calli. For grape cell control, the same size of PDA discs without fungal mycelia were inoculated. The dual cultures and controls were dual cultured continuously in dark conditions in an oven at $25^{\circ} \mathrm{C}$ for 5 days. Every treatment and control included at least 5 biological replicates. Grape cells undergone different treatments were harvested for transcriptomic and metabolomics assay.As for fungal extracts treatments, the fungal extracts were diluted to a fresh fungal mycelium concentration equivalent to $10 \mu \mathrm{g} / \mathrm{mL}$, respectively. The solutions with different concentrations of mycelial extracts were filter sterilized, and a $0.1 \mathrm{~mL}$ aliquot was evenly transferred to the medium surface of each B5 agar plate. The same volume of sterilized distilled water was added to B5 agar plates as a control. Approximately $1.5 \mathrm{~g}$ of prepared grape calli was then inoculated into the center of each plate and cultured for 5 days also in dark conditions in an oven at $25{ }^{\circ} \mathrm{C}$. Grape calli treated with different fungal extracts and the controls were harvested and also sent to transcriptome and metabolome analysis.

RNA sequencing: The total RNA was extracted from frozen grape cell samples, and the mRNA library of each sample was constructed and sequenced in the Illumina HiSeq4000 platform. The adaptor and low- 
quality sequence were removed using Fastp with default parameters (Chen, Zhou, Chen, \& Jia, 2018), and clean reads were then mapped to the grapevine $12 \times$ genome assembly available from EnsemblPlants (http://plants.ensembl.org/Vitis_vinifera/Info/Index), using HISAT2 [18]. Quantification of gene expression levels were estimated by fragments per kilobase of transcript per million fragments mapped (FPKM). The formula is shown as follow: FPKM = cDNA Fragments/[Mapped Fragments (Millions) $\times$ Transcript Length $(\mathrm{kb})$ ]. Transcripts profiling were carried for 3 of all prepared biological replicates. Based on the raw count data, differential expression analysis between samples was performed by DESeq2 software [19]. Genes satisfying |log2Fold Change| $>=0$, and padj $<0.05$ were defined as differentially expressed genes (DEGs).

Metabolite profiling: Metabolite profiling was carried out using a widely targeted metabolome method by Wuhan Metware Biotechnology Co., Ltd. (Wuhan, China) (http://www.metware.cn/). The freeze-dried samples were extracted as described previously [20]. The extracts were analyzed using an LC-ESI-MS/MS system (UPLC, Shim-pack UFLC SHIMADZU CBM30A system; MS, Applied Biosystems 6500 Q TRAP). Metabolite quantification was performed using a scheduled multiple reaction monitoring (MRM) method, which has been described [20]. Metabolites profiling were carried for 3 of all prepared biological replicates. The identified metabolites were subjected to orthogonal partial least squares discriminant analysis (OPLS-DA), and metabolites with $\mid$ Log2 (fold change)| > = 0, and VIP』variable importance in project $\backslash>=1$ were considered as differentially regulated metabolites (DRMs).

Statistical Data Analysis: Genes satisfied the threshold of |log2Fold Change| $>=0$, and padj $<0.05$ were considered as significant DEGs and subjected to Gene Ontology (GO) and Kyoto Encyclopedia of Genes and Genomes (KEGG) enrichment analysis. In metabolome analysis, significant DRMs between groups were determined by VIP $>=1$ and absolute Log2FC (fold change) $>=1$. VIP values were extracted from OPLS-DA result, which also contain score plots and permutation plots, was generated using $\mathrm{R}$ package ropls. The data was log transform (log2) and pare to-scaling before OPLS-DA. In order to avoid overfitting, a permutation test (200 permutations) was performed. The significant enriched $\mathrm{GO}$ terms and KEGG pathways were defined as $-\log 10$ (padj > = 1.5. Figures were generated using Sigma Plot 12.5 (Systat Software Inc., San Jose, CA) and R- Program.

\section{Declarations}

\section{Ethics approval and consent to participate}

Not applicable

\section{Consent for publication}

Not applicable

\section{Availability of data and materials}


All data generated or analyzed during this study are included in this published article [and its supplementary information files].

\section{Competing Interests}

The authors declare that they have no competing interests.

\section{Finding}

This work was financially supported by the National Natural Science Foundation of China (NSFC: 31160070) \And the joint foundation of Yunnan Provincial department of Science and Technology and Yunnan University (No. 2019FY003024).

\section{Authors contributions:}

$M Z$, planed and designed the researches, guided the performance of experiments, wrote the main manuscript; JZ, performed most of the experiments and raw data acquisitions; HB, participated the figures preparation and manuscript editing; LF, XX, YM, ZM, SY, CM and LT participated partially or assisted the experiments and data analysis. All authors have read and approved the manuscript", and ensure that this is the case.

\section{Acknowledgements}

Not applicable

\section{References}

1. Hardoim, P.R., et al., The Hidden World within Plants: Ecological and Evolutionary Considerations for Defining Functioning of Microbial Endophytes. Microbiology \& Molecular Biology Reviews, 2015. 79(3): p. 293-320.

2. Pacifico, D., et al., The Role of the Endophytic Microbiome in the Grapevine Response to Environmental Triggers. Frontiers in plant science, 2019. 1256(10): p. 15.

3. Fernandez, O., et al., Trehalose metabolism is activated upon chilling in grapevine and might participate inBurkholderia phytofirmansinduced chilling tolerance. Planta, 2012. 236(2): p. 355-369.

4. Qassim, et al., Paraburkholderia phytofirmans PsJN-Plants Interaction: From Perception to the Induced Mechanisms. Frontiers in microbiology, 2018. 2093(9).

5. Musetti, R., et al., Inhibition of Sporulation and UItrastructural Alterations of Grapevine Downy Mildew by the Endophytic Fungus ||$r<i>A /$ ternaria alternata</i>. Phytopathology, 2006. 96(7): p. 689-698.

6. Yang, M.-Z., et al., Fungal Endophytes as a Metabolic Fine-Tuning Regulator for Wine Grape. PLoS ONE, 2016. 11(9): p. e0163186.

7. Huang, L.H., et al., Endophytic fungi specifically introduce novel metabolites into grape flesh cells in vitro. Plos One, 2018. 13(5): p. e0196996. 
8. Cordovez., V., et al., Ecology and Evolution of Plant Microbiomes. Annual Review of Microbiology, 2019. 73(1): p. 19.

9. Yang, M.-Z., et al., Endophytic Fungal strains Specifically Modified the Biochemical Status of Grape Cells. Journal of Plant Biology, 2018. 61: p. 7.

10. Huang, L.-h., et al., In vitro specific interactions revealed the infective characteristics of fungal endophytes to grapevine. Vitis, 2017. 56: p. 71-77.

11. Zhu, B., et al., Fungal elicitors stimulate biomass and active ingredients accumulation in Dendrobium catenatum plantlets. Biologia, 2018. 73: p. 917-926.

12. Senda, K., N. Doke, and K. Kawakita, Effect of Mastoparan on Phospholipase A2 Activity in Potato Tubers Treated with Fungal Elicitor. Plant and Cell Physiology, 1998. 39(10): p. 1080-1098.

13. Zhang, R., et al., Enhancement of Diosgenin Production in Dioscorea zingiberensis Cell Culture by Oligosaccharide Elicitor from its Endophytic Fungus Fusarium oxysporum Dzf17. 2009. 4(11): p. 1459.

14. Yu, M., et al., Exposure to endophytic fungi quantitatively and compositionally alters anthocyanins in grape cells. Plant Physiology and Biochemistry, 2020. 149: p. 8.

15. Gao, F.-K., C.-G. Ren, and C.-C. Dai, Signaling effects of nitric oxide, salicylic acid, and reactive oxygen species on isoeuphpekinensin accumulation in Euphorbia pekinensis suspension cells induced by an endophytic fungal elicitor. Journal of plant growth regulation, 2012. 31(4): p. 490-497.

16. Li, P., et al., Effects of polysaccharide elicitors from endophytic Fusarium oxysporium Dzf17 on growth and diosgenin production in cell suspension culture of Dioscorea zingiberensis. Molecules, 2011. 16(11): p. 9003-9016.

17. Wang, J.W., Z. Zhang, and R.X. Tan, Stimulation of artemisinin production in Artemisia annua hairy roots by the elicitor from the endophytic Colletotrichum sp. Biotechnology letters, 2001. 23(11): p. 857-860.

18. Kim, D., B. Langmead, and S.L. Salzberg, HISAT: a fast spliced aligner with low memory requirements. Nature Methods, 2015. 12(4): p. 357-360.

19. Varet, H., et al., SARTools: A DESeq2- and EdgeR-Based R Pipeline for Comprehensive Differential Analysis of RNA-Seq Data. Plos One, 2016. 11.

20. Chen, W., et al., A Novel Integrated Method for Large-Scale Detection, Identification, and Quantification of Widely Targeted Metabolites: Application in the Study of Rice Metabolomics. Molecular Plant, 2013. 6(6): p. 1769-1780.

\section{Figures}



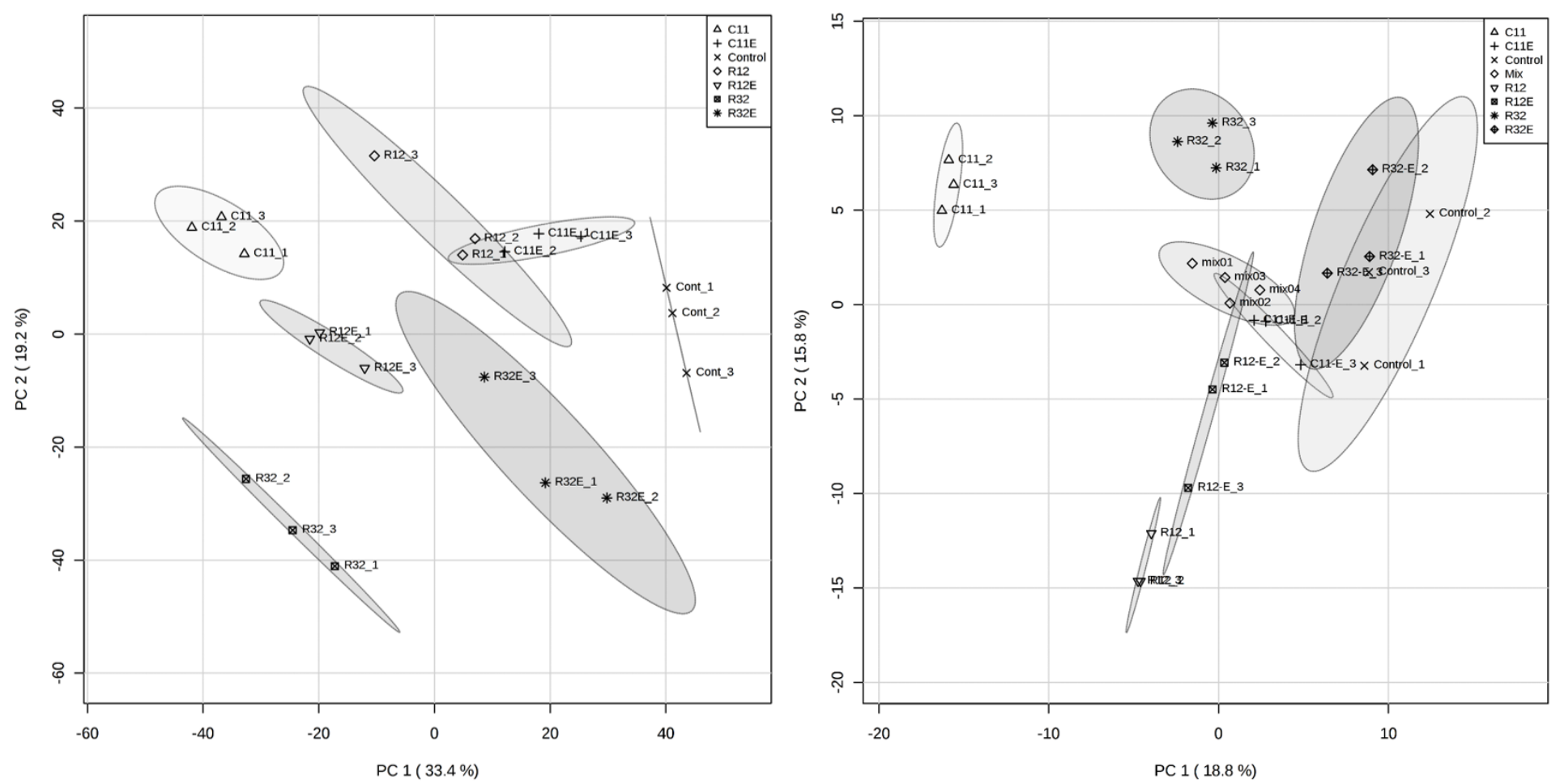

Figure 1

Principal components analysis (PCA) to the transcripts (left) and metabolites (right) profiles in treated grape cells. PCAs were performed by R program based on the transcriptome and metabolome patterns of grape cells after treated with different endophytic fungal strains and their corresponding soluble extracts.
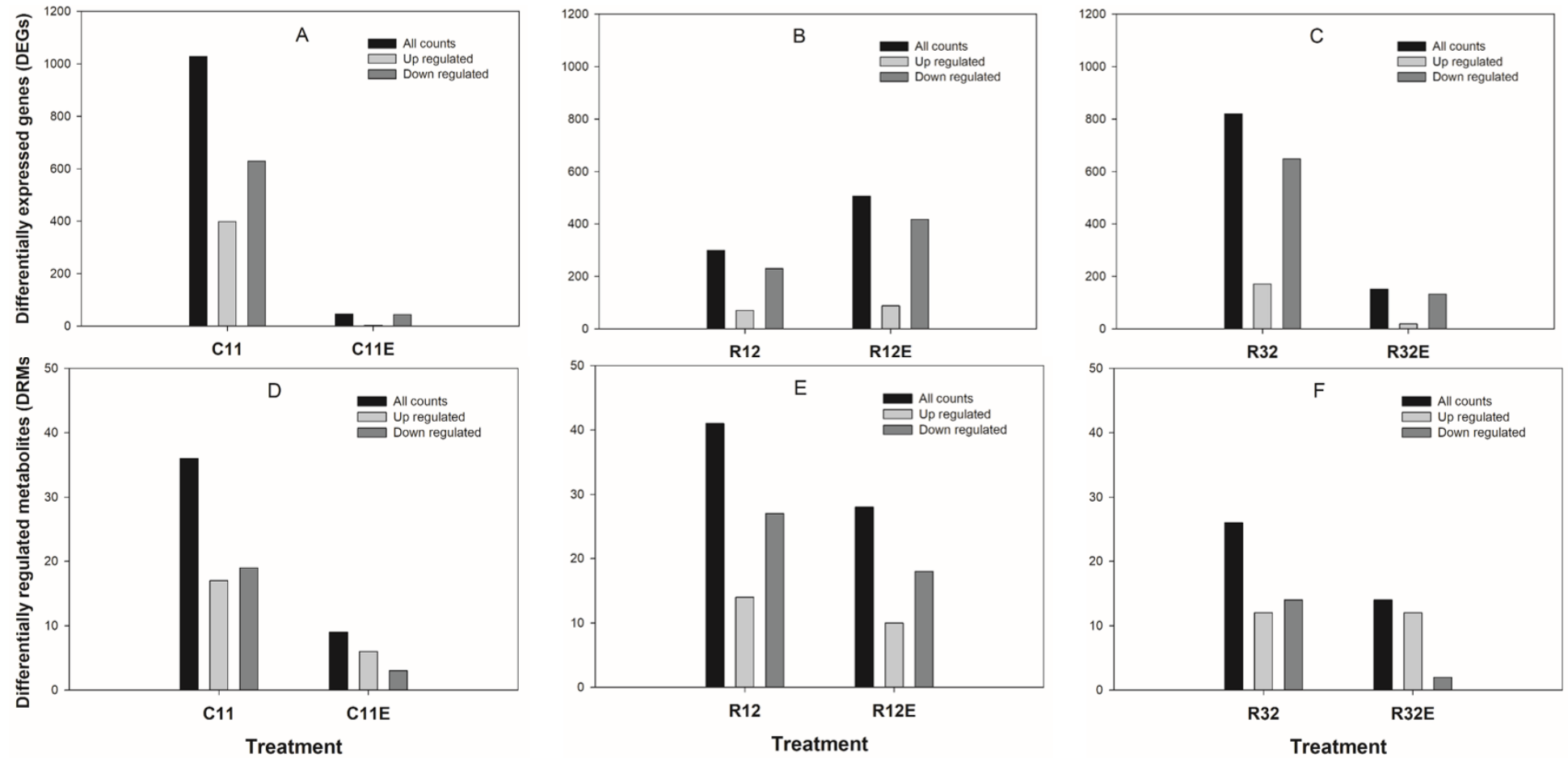

Figure 2 
Counts of differentially expressed genes (DEGs) (Up) and differentially regulated metabolites (DRMs) (Below) in different treated grape cells. DEGs and DRMs were calculated by comparing to the corresponding controls.
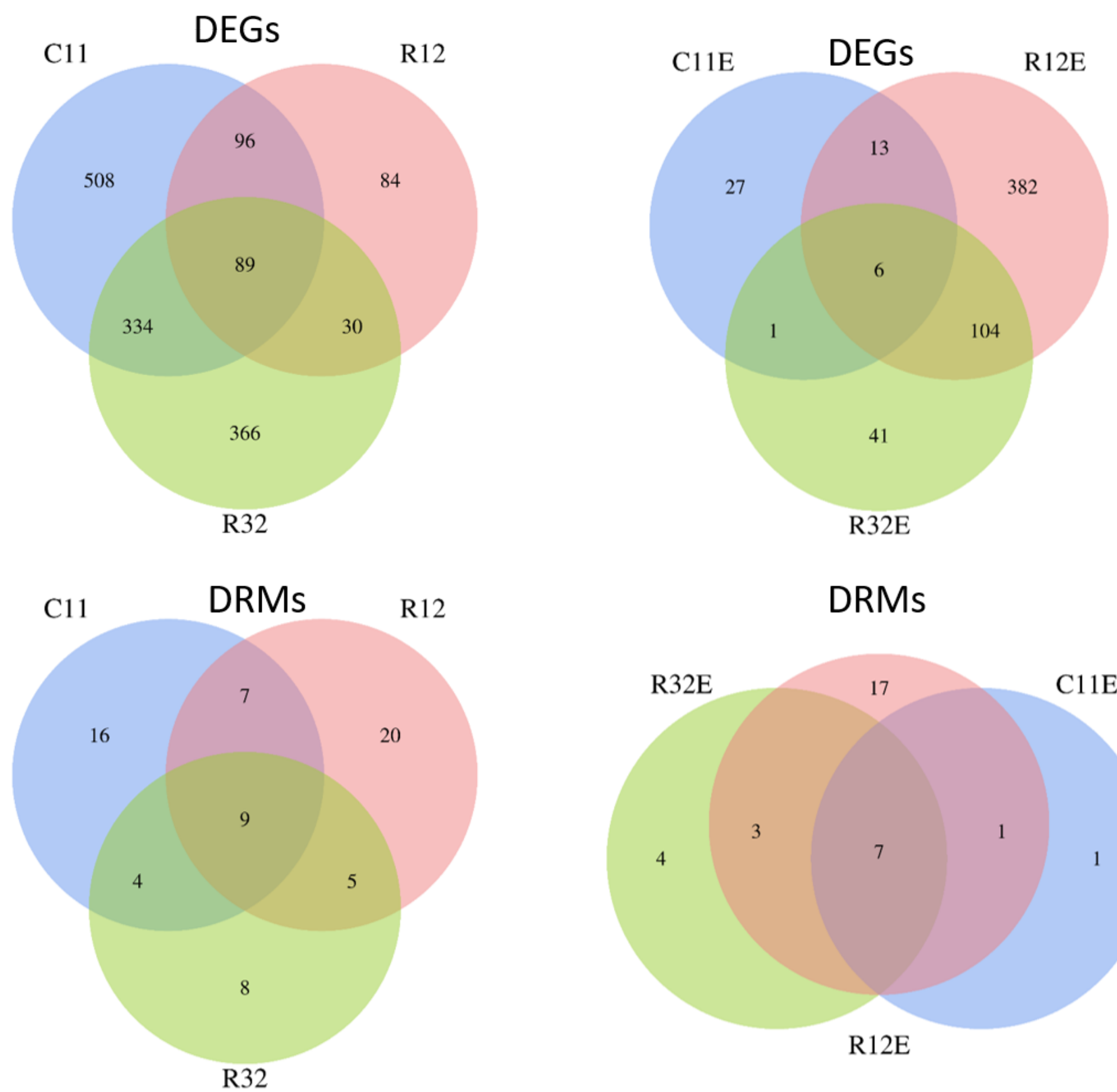

\section{DRMs}

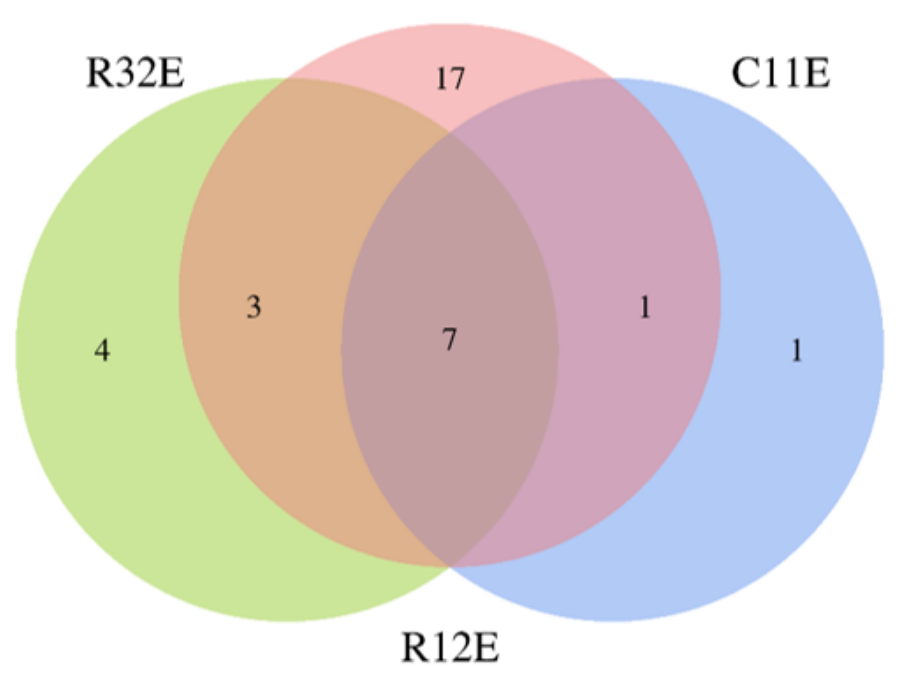

\section{Figure 3}

Venn plots for displaying the co-detected and specific counts of DEGs and DRMs in grape cells after the exposures to different fungal strains and fungal extracts, respectively. 


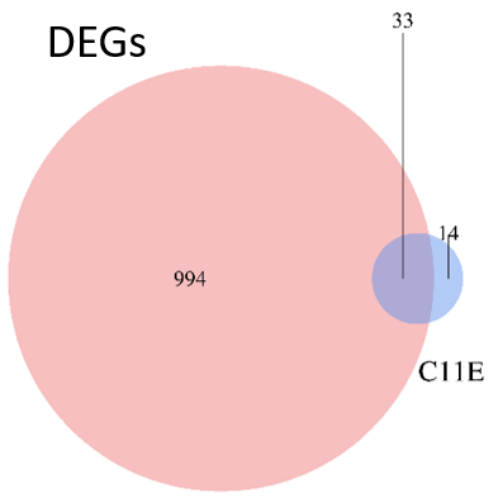

$\mathrm{C} 11$

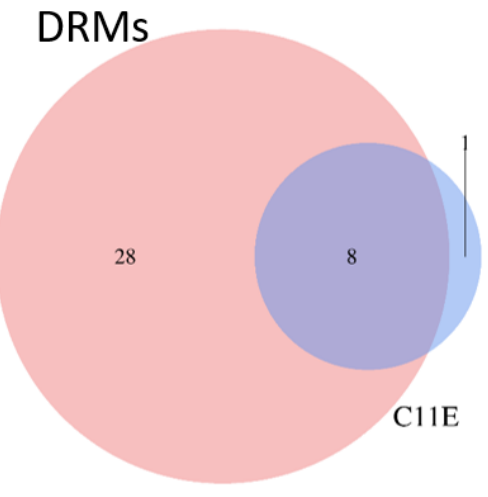

$\mathrm{C} 11$
DEGs

R12E

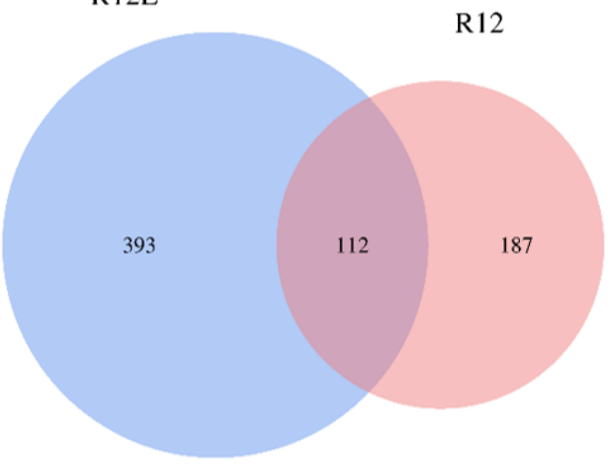

DRMs

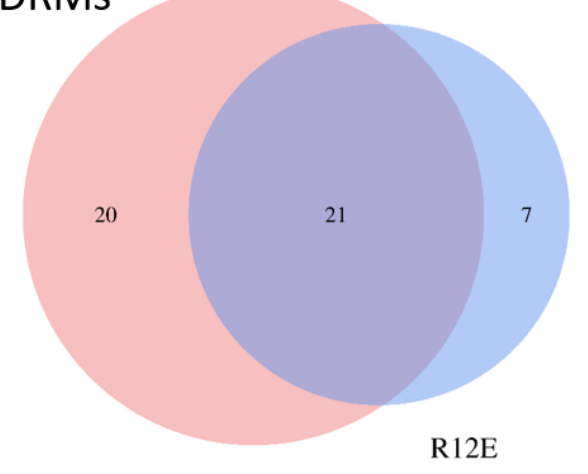

R12
DEGs

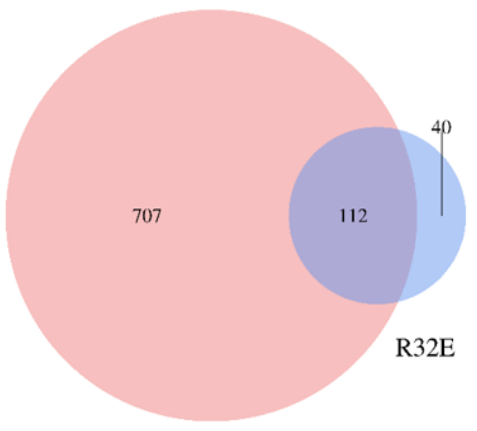

R32

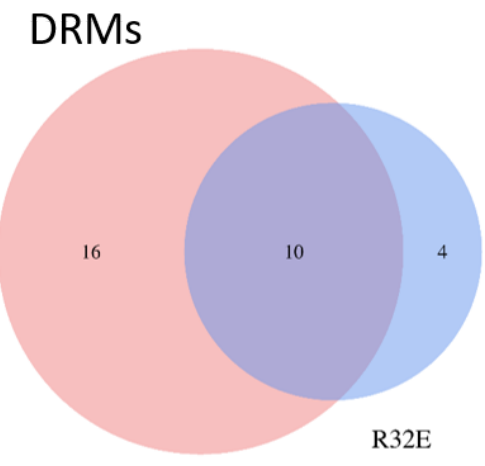

$\mathrm{R} 32$

\section{Figure 4}

Venn plots for displaying the co-detected or specific counts of DEGs and DRMs between grape cells treated with living fungi and the corresponding fungal extracts. 

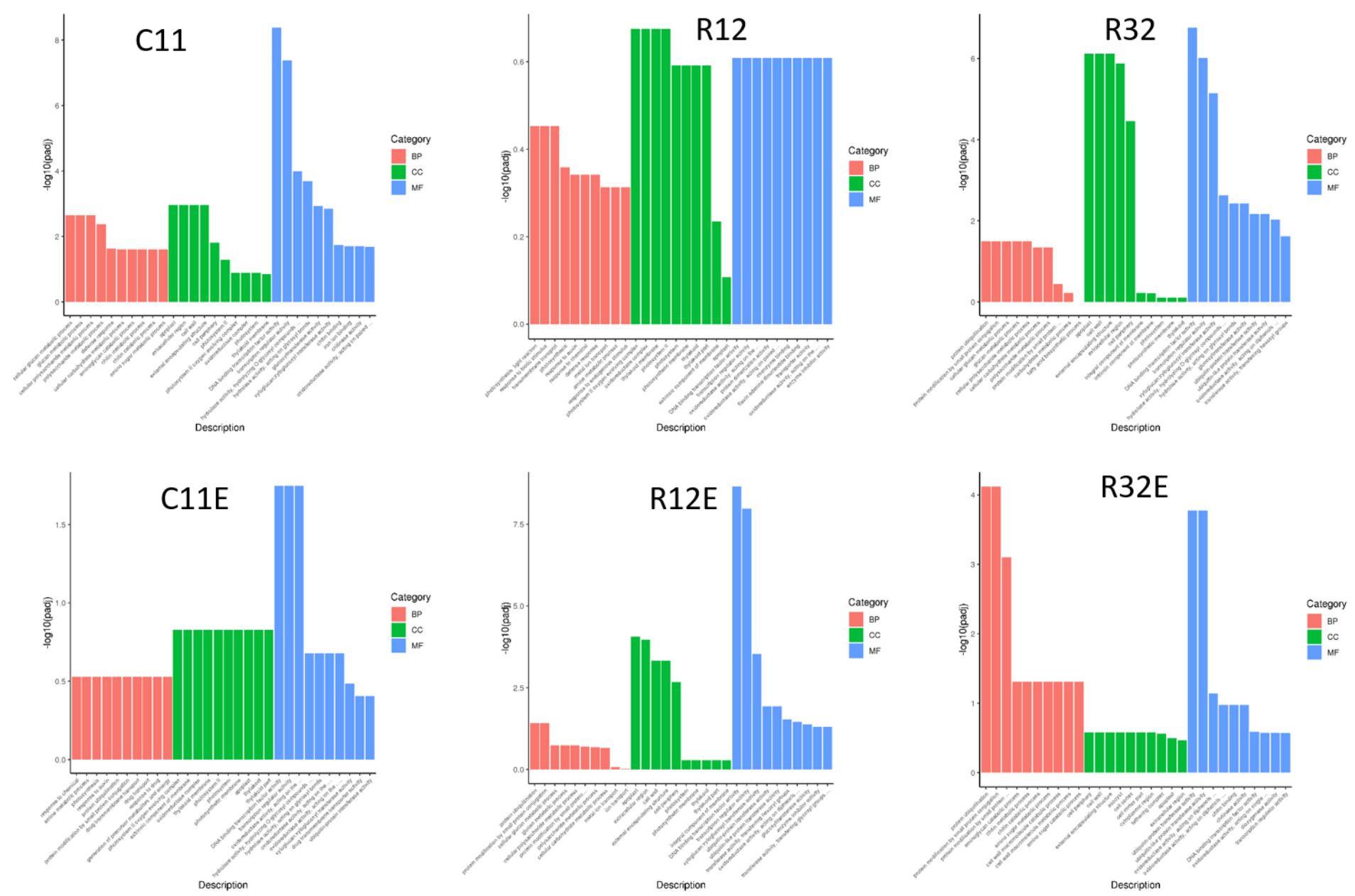

Figure 5

The most (top 30) enriched Gene ontology (GO) terms in grape cells treated with different living fungi and fungal extracts. 

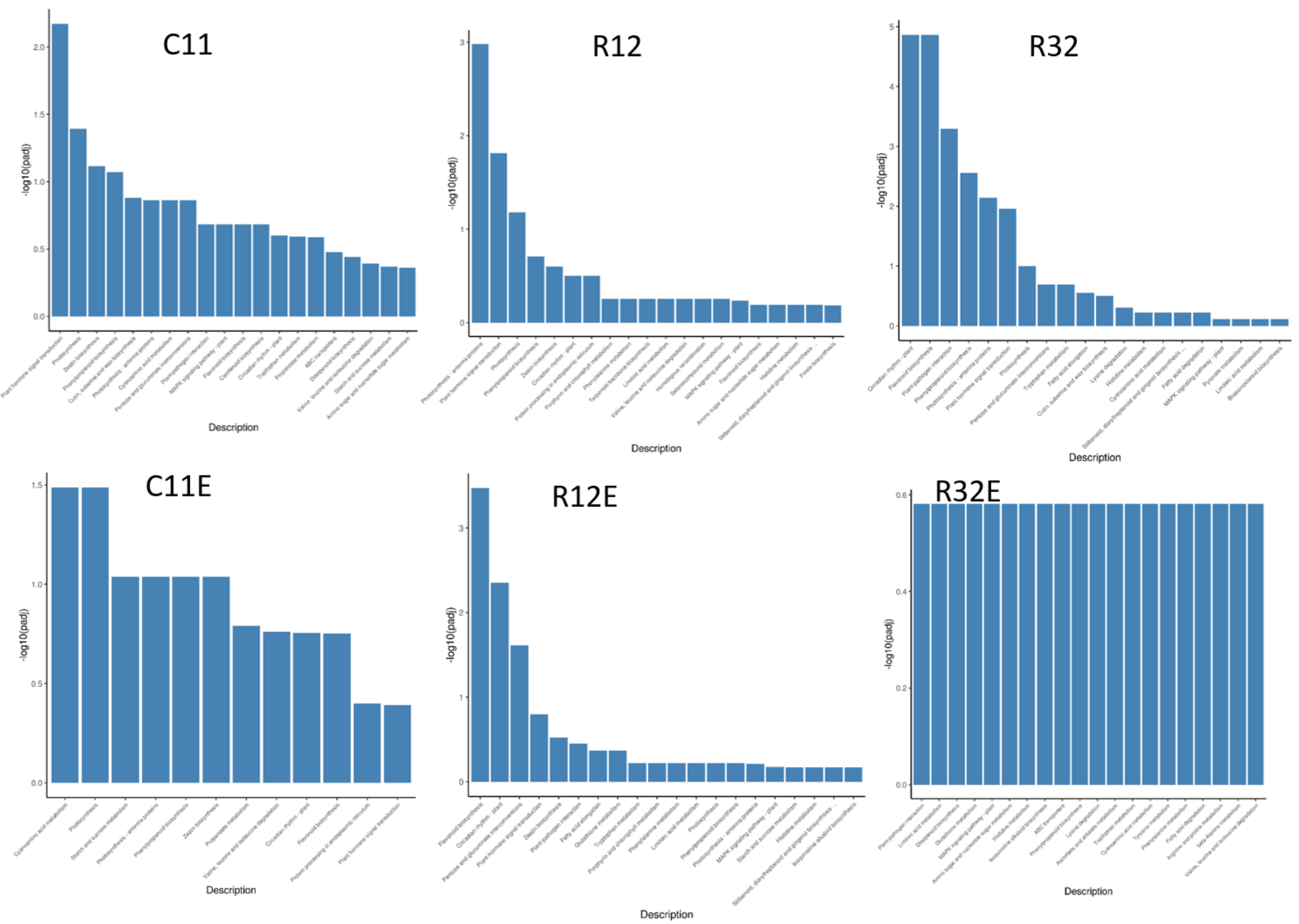

Figure 6

Bar plots of the top 20 most enriched KEGG pathways in different living fungi and fungal extracts exposed grape cells. 
Enriched GO terms

C11E

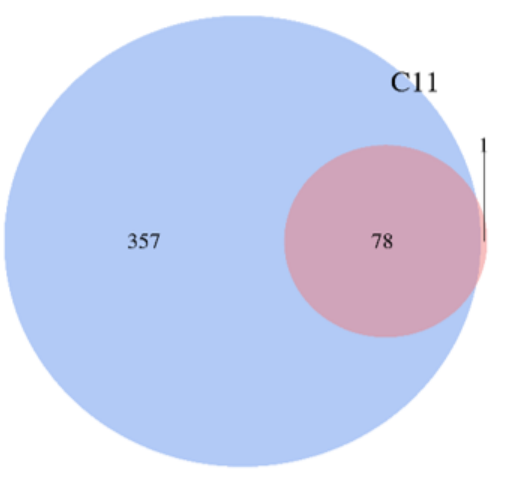

Enriched KEGG pathways C11

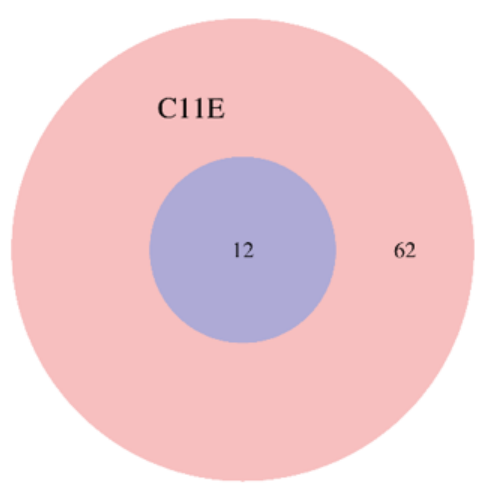

Enriched GO terms

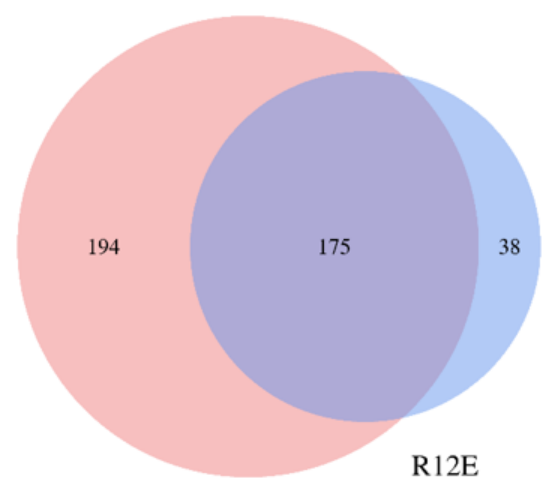

R12

Enriched KEGG pathways

R12

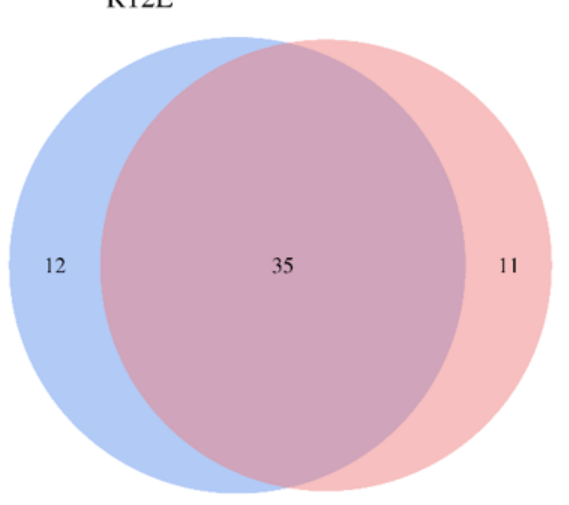

Enriched GO terms

R32

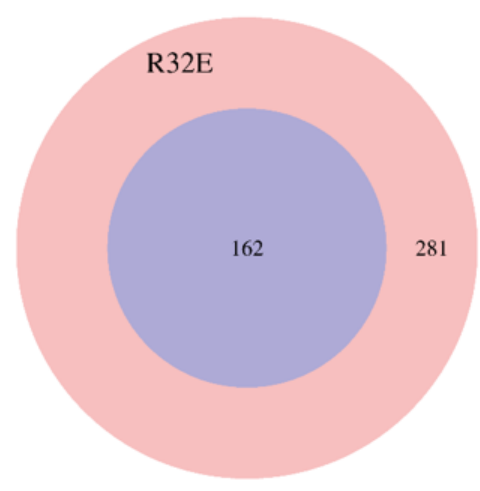

Enriched KEGG pathways R32

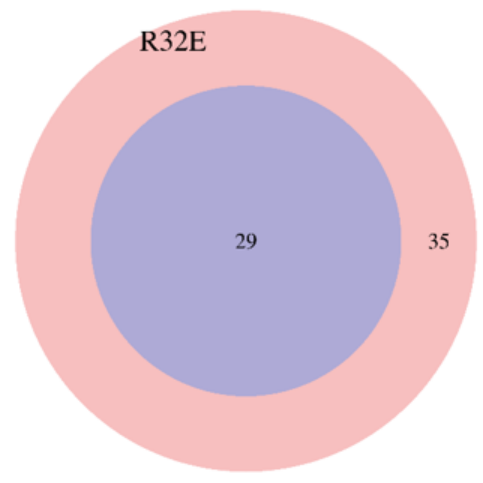

Figure 7

Venn plots displayed the co-enriched and specific GO terms (up) and KEGG pathways (below) between living fungi and the corresponding fungal extracts exposed grape cells. 

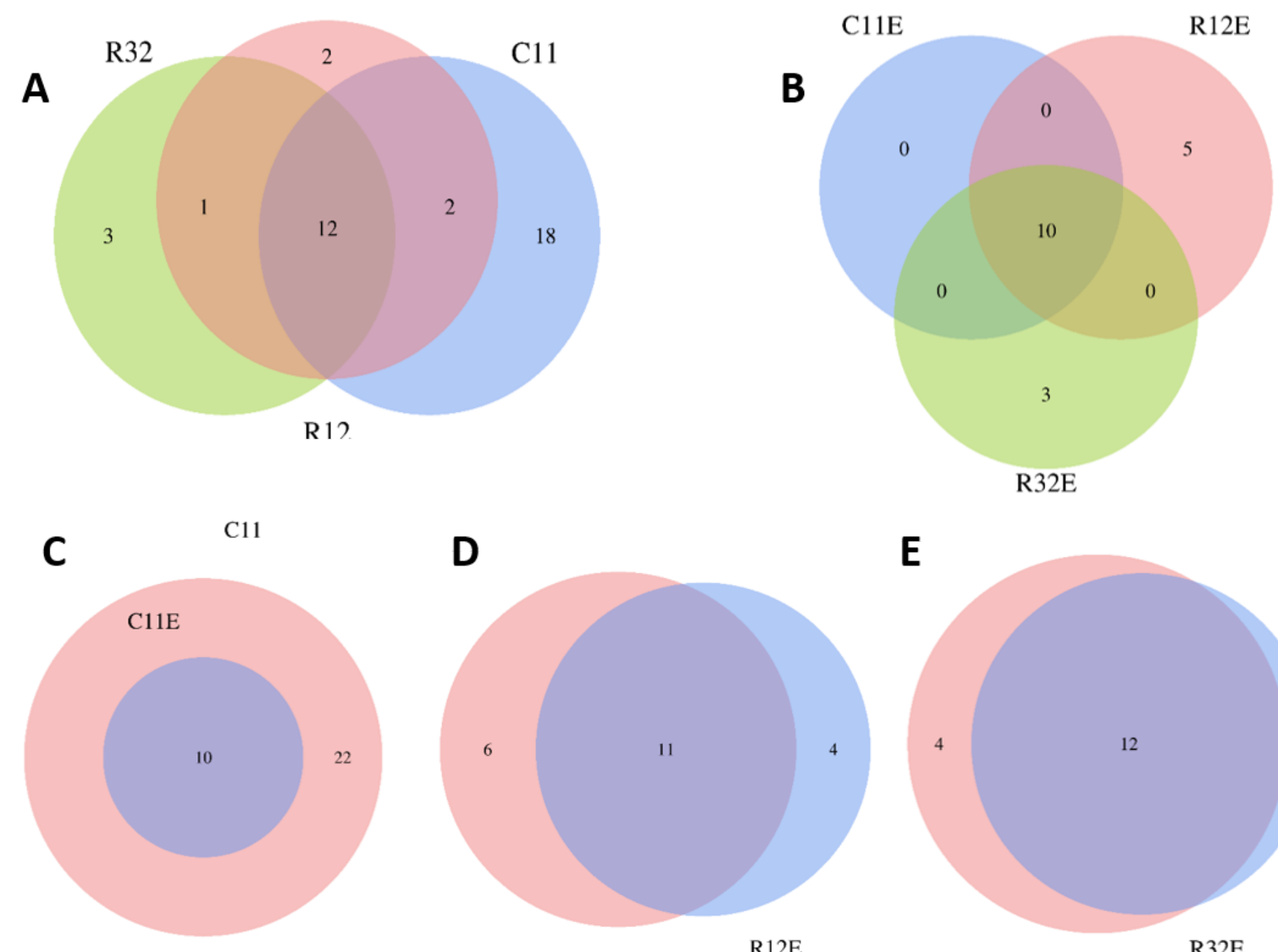

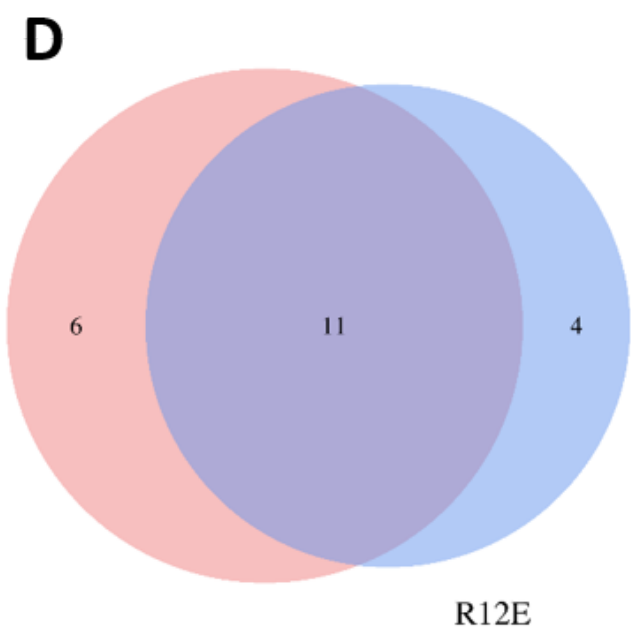

R32

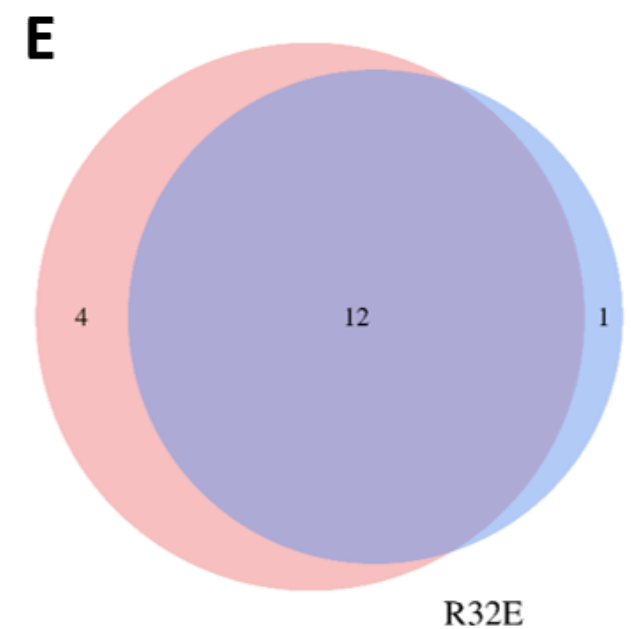

R32

\section{Figure 8}

Venn plots displayed the co-enriched or specific KEGG pathways among multiple treated grape cells. The co-enriched or specific KEGG pathways among different living fungi (A) and different fungal extracts (B) treated grape cells, as well as between the living fungi and the corresponding fungal extracts treated grape cells (C, D and E).

\section{Supplementary Files}

This is a list of supplementary files associated with this preprint. Click to download.

- Additionalfile1QCdatatables.pdf

- additionalfile3METABOLOMEDATASET.pdf

- additionalfile2Transcriptomedataset.pdf 